\title{
Corrigendum: Intensification of the meridional temperature gradient in the Great Barrier Reef following the Last Glacial Maximum
}

Thomas Felis, Helen V. McGregor, Braddock K. Linsley, Alexander W. Tudhope, Michael K. Gagan, Atsushi Suzuki, Mayuri Inoue, Alexander L. Thomas, Tezer M. Esat, William G. Thompson, Manish Tiwari, Donald C. Potts, Manfred Mudelsee, Yusuke Yokoyama \& Jody M. Webster

Nature Communications 5:4102 doi: 10.1038/ncomms5102 (2014); Published 17 Jun 2014; Updated 15 Jun 2016

The original version of this Article failed to fully credit the use of the Ocean Data View software in figure 1 and supplementary figures 6 and 7, which appears below:

Schlitzer, R., Ocean Data View, http://odv.awi.de, 2016. 